Int. J. Electrochem. Sci., 16 (2021) Article ID: 210326

\title{
Electrochemical-thermal coupled model for the optimal design of a liquid cooling module of a cylindrical lithium-ion battery
}

\author{
Huanhuan Li ${ }^{1,3}$, Yijie Wang ${ }^{1}$, Zhengjian $\mathrm{Gu}^{4}$, Yaping Wang, ${ }^{2,3,}$, Chaofeng Pan ${ }^{1}$, Long Chen ${ }^{1}$, \\ Haobin Jiang ${ }^{l}$ \\ ${ }^{1}$ Automotive Engineering Research Institute, Jiangsu University, 301 Xuefu Road, Zhenjiang 212013, \\ P. R. China \\ ${ }^{2}$ School of Materials Science \& Engineering, Jiangsu University, Zhenjiang 212013, P. R. China \\ ${ }^{3}$ Key Laboratory of Advanced Energy Materials Chemistry (Ministry of Education), College of \\ Chemistry, Nankai University, 94 Weijing Road, Tianjin, 300071, PR China \\ ${ }^{4}$ Advanced Energy Storage Products Quality Supervision and Inspection Center, 8 Chunxin East Road, \\ Wuxi, 214101, P. R. China \\ *E-mail: wangyaping@ujs.edu.cn
}

doi: $10.20964 / 2021.03 .35$

Received: 3 November 2020 / Accepted: 22 December 2020 / Published: 31 January 2021

To enable a battery to operate in a suitable temperature range, a three-dimensional electrochemicalthermal fully coupled model of a cylindrical lithium-ion battery (LIB) including a spiral wound structure is constructed. The precise temperature distribution and transient heat generation rate inside the battery are obtained by considering charge conservation, mass transfer, energy conservation and electrode dynamics processes. Based on the constructed model, a semi-enclosed S-shaped surrounding structure is proposed to dissipate the heat of the battery module. The effects of cooling channel height, width and inlet velocity on the heat dissipation performance are studied. The height of the cooling channel is close to the height of the active material, which can affect both the cooling performance and portability of the liquid cooling device. Meanwhile, when the width of the liquid cooling channel is $3 \mathrm{~mm}$, it not only has the best cooling effect but also improves the space utilization, and when the inlet velocity is more than $0.15 \mathrm{~m} / \mathrm{s}$, the maximum temperature and temperature difference of the module during the entire discharge process can be kept under $303 \mathrm{~K}$ and $5 \mathrm{~K}$. In addition, by considering the thermal conductivity differences between the axial and radial directions of the active material, a decentralized optimization design of multiple cooling channels in the vertical direction is proposed. The results show that a structure with three channels in the vertical direction and a height of the middle channel twice that of the upper and lower ones is more favourable for heat transmission from the inside to the outside because it disperses the heat dissipation surface area to improve the temperature consistency of the module and effectively improves the heat dissipation performance of the liquid cooling structure. 
Keywords: Lithium-ion battery; Electrochemical-thermal coupled; Liquid cooling; Structural optimization

\section{FULL TEXT}

(C) 2021 The Authors. Published by ESG (www.electrochemsci.org). This article is an open access article distributed under the terms and conditions of the Creative Commons Attribution license (http://creativecommons.org/licenses/by/4.0/). 